\title{
Fatal Outcomes of COVID-19 in Patients with Severe Acute Kidney Injury
}

\author{
Jeong-Hoon Lim ${ }^{1,+}{ }^{\circledR}$, Sun-Hee Park ${ }^{1,+}$, Yena Jeon ${ }^{2}$, Jang-Hee Cho ${ }^{1}{ }^{\circledR}$, Hee-Yeon Jung ${ }^{1}{ }^{(}$, \\ Ji-Young Choi ${ }^{1}{ }^{1}$, Chan-Duck Kim ${ }^{1}$, Yong-Hoon Lee ${ }^{3}$, Hyewon Seo ${ }^{3}$, Jaehee Lee ${ }^{3}$, \\ Ki Tae Kwon ${ }^{4}$, Shin-Woo Kim ${ }^{4}$, Hyun-Ha Chang ${ }^{4, *}$ and Yong-Lim Kim ${ }^{1, *}$ \\ 1 Division of Nephrology, Department of Internal Medicine, School of Medicine, \\ Kyungpook National University, Daegu 41944, Korea; jh-lim@knu.ac.kr (J.-H.L.); \\ sh-park@knu.ac.kr (S.-H.P.); jh-cho@knu.ac.kr (J.-H.C.); hy-jung@knu.ac.kr (H.-Y.J.); \\ jyss1002@hanmail.net (J.-Y.C.); drcdkim@knu.ac.kr (C.-D.K.) \\ 2 Department of Statistics, Kyungpook National University, Daegu 41566, Korea; yeahnah@naver.com \\ 3 Division of Pulmonology and Critical Care Medicine, Department of Internal Medicine, School of Medicine, \\ Kyungpook National University, Daegu 41944, Korea; id0121@naver.com (Y.-H.L.); \\ dallyu17@hanmail.net (H.S.); jaelee@knu.ac.kr (J.L.) \\ 4 Division of Infectious Disease, Department of Internal Medicine, School of Medicine, Kyungpook National \\ University, Daegu 41944, Korea; idktkwon@gmail.com (K.T.K.); ksw2kms@knu.ac.kr (S.-W.K.) \\ * Correspondence: changhha@knu.ac.kr (H.-H.C.); ylkim@knu.ac.kr (Y.-L.K.); \\ Tel.: +82-53-200-6553 (H.-H.C.); +82-53-200-5553 (Y.-L.K.) \\ + These two authors contributed equally to this work.
}

Received: 1 May 2020; Accepted: 27 May 2020; Published: 3 June 2020

check for updates

\begin{abstract}
The outcome of coronavirus disease 2019 (COVID-19) is associated with organ damage; however, the information about the relationship between acute kidney injury (AKI) and COVID-19 is still rare. We evaluated the clinical features and prognosis of COVID-19 patients with AKI according to the AKI severity. Medical data of hospitalized COVID-19 patients in two university-based hospitals during an outbreak in Daegu, South Korea, were retrospectively analyzed. AKI and its severity were defined according to the Acute Kidney Injury Network. Of the 164 hospitalized patients with COVID-19, 30 patients (18.3\%) had AKI; 14, 4, and 12 patients had stage 1, 2, and 3, respectively. The median age was significantly higher in AKI patients than in non-AKI patients (75.5 vs. 67.0 years, $p=0.005)$. There were 17 deaths (56.7\%) among AKI patients; $4(28.6 \%), 1(25.0 \%)$, and $12(100.0 \%)$, respectively. In-hospital mortality was higher in AKI patients than in non-AKI patients (56.7\% vs. $20.8 \%, p<0.001)$. After adjusting for potential confounding factors, stage 3 AKI was associated with higher mortality than either non-AKI or stage $1 \mathrm{AKI}$ (hazard ratio $(\mathrm{HR})=3.62(95 \%$ confidence interval $(\mathrm{CI})=1.75-7.48), p=0.001 ; \mathrm{HR}=15.65(95 \% \mathrm{CI}=2.43-100.64), p=0.004)$. Among the AKI patients, acute respiratory distress syndrome and low serum albumin on admission were considered independent risk factors for stage 3 AKI (both $p<0.05$ ). Five patients with stage 3 AKI underwent dialysis and eventually died. In conclusion, COVID-19 patients with severe AKI had fatal outcomes.
\end{abstract}

Keywords: acute kidney injury; AKI severity; COVID-19; mortality; renal replacement therapy

\section{Introduction}

The new coronavirus, named severe acute respiratory syndrome coronavirus 2 (SARS-CoV-2), which originated in Wuhan, China, in December 2019 is spreading rapidly worldwide [1-3]. The World Health Organization declared the coronavirus disease 2019 (COVID-19) pandemic on 11 March 2020 and countries around the world are trying to prevent the spread of this virus [4]. However, the spread of infection is continuing and there is a lack of information on how to cope with it. 
In February 2020, the new coronavirus spread rapidly to the community through a group worship session in a religious group named "Shincheonji" in Daegu, South Korea. The new coronavirus has a lower fatality rate but a higher infection rate than severe acute respiratory syndrome coronavirus (SARS-CoV) and Middle East respiratory syndrome coronavirus (MERS-CoV), which caused previous epidemics [5]. In particular, patients with underlying diseases, such as diabetes, hypertension, and cardiovascular disease, as well as older patients, are more likely to become infected and have a higher risk of progression to a severe condition [6]. Moreover, the mortality rate is high when accompanied by organ dysfunctions, such as in the kidneys and lungs [7].

The close relationship between acute kidney injury (AKI) and coronavirus infection has previously been identified in SARS-CoV and MERS-CoV epidemics. The reported incidence of AKI in SARS-CoV was $6.7 \%$, whereas the AKI prevalence was up to $43.0 \%$ in MERS, which had a higher fatality than SARS-CoV [8]. Patients with severe infection, comorbid disease, and organ failure were associated with AKI occurrence, and mortality was high in these AKI patients (SARS-CoV, 91.7\%; MERS-CoV, $70.0 \%)[9,10]$.

Regarding COVID-19, there is limited information on accompanying AKI; therefore, we examined the clinical course and prognosis of patients according to AKI severity. This study aimed to share the clinical features and treatment experience of COVID-19 patients with AKI.

\section{Materials and Methods}

\subsection{Patients and Data Collection}

In South Korea, COVID-19 patients who are asymptomatic and with mild severity were quarantined in therapeutic living centers assigned by the government to cope with a large number of patients and the hospital-bed shortage [11]. Patients with moderate severity were hospitalized in community hospitals, whereas severe and critical patients were admitted to designated COVID-19 treatment tertiary hospitals. We classified COVID-19 patients according to a brief telephone severity scoring system into four categories: asymptomatic to mild, moderate, severe, and critical [11]. The Kyungpook National University Hospital and Kyungpook National University Chilgok Hospital located in Daegu, South Korea, were designated as professional COVID-19 treatment tertiary hospitals for the treatment of patients of severe to critical severity. All COVID-19 patients who were admitted to these two hospitals from 17 February to 22 March 2020 were retrospectively reviewed; patient data were collected until 15 May 2020. All COVID-19 patients who had AKI during inpatient treatment were registered in this study, except those with underlying chronic kidney disease (CKD, defined as an estimated glomerular filtration rate (eGFR) of $<60 \mathrm{~mL} / \mathrm{min} / 1.73 \mathrm{~m}^{2}$ ). Underlying CKD was identified using previous medical records, laboratory data, and history taking. Data on patient demographics, clinical symptoms, comorbid conditions, clinical course, and laboratory data were collected from electronic medical records. The laboratory data set included complete blood counts, liver function tests, renal function tests, and inflammatory markers. eGFR was calculated using the Chronic Kidney Disease Epidemiology Collaboration (CKD-EPI) equation [12]. The study protocol was reviewed and approved by the institutional review boards of Kyungpook National University Hospital (2020-04-059) and Kyungpook National University Chilgok Hospital (2020-04-013). The requirement for informed consent was waived because this study did not infringe on the privacy or health of the patients. All patient data were anonymized and de-identified before the analyses took place.

\subsection{Virologic Studies}

The diagnosis of COVID-19 was made through nasopharyngeal and oropharyngeal swab samples using a real-time reverse transcription polymerase chain reaction (rRT-PCR) for SARS-CoV-2. The collected samples were put into a collection tube with $150 \mu \mathrm{L}$ of viral preservation solution and RNA was extracted with an Allplex 2019-nCoV assay (Seegen, Seoul, Korea), per the manufacturer's instructions. Three target genes for SARS-CoV-2 were 
amplified and tested during each rRT-PCR assay. Detailed information for the target gene detection are as follows: RNA-dependent RNA polymerase (RdRp) gene: forward primer, 5'-GTGARATGGTCATGTGTGGCGG-3'; reverse primer, 5' -CARATGTTAAASACACTATTAGC ATA-3'; and probe in the 5-FAM/3'-BHQ format, 5' ${ }^{\prime}$-CAGGTGGAACCTCATCAGGAGATGC- $3^{\prime}$; E gene: forward primer, 5'-ACAGGTACGTTAATAGTTAATAGCGT-3'; reverse primer, $5^{\prime}$-ATATTGCAGCAGTACGCAC ACA-3'; and probe in the 5-FAM/3'-BHQ format, 5'-ACACTAGCCATCCTTACTGCGCTTCG-3'; $\mathrm{N}$ gene: forward primer, 5'-CACATTGGCACCCGCAATC-3'; reverse primer, 5'-GAGGAACGAGAAGAGGCTTG-3'; and probe in the 5-FAM/3'-BHQ format, 5'-ACTTCCTCAAGGAACAACATTGCCA-3'.

The RNA copy numbers were calculated using a standard curve based on the cycle threshold $(\mathrm{Ct})$ values of plasmid DNA. Each dilution of plasmid DNA was tested in duplicate to produce the standard curve. The SARS-CoV-2 RNA copy numbers were converted from the $\mathrm{Ct}$ values of rRT-PCR. The result was considered positive if the $\mathrm{Ct}$ values were $<37$ cycles; the limit of detection was 100 copies $/ \mu \mathrm{L}$.

\subsection{Definition}

We defined AKI using the definition of the Acute Kidney Injury Network (AKIN): (a) an increase in the serum creatinine level to $\geq 0.3 \mathrm{mg} / \mathrm{dL}$, (b) an increase in baseline serum creatinine level to $\geq 150 \%$, or (c) the initiation of dialysis without a history of CKD [13]. The baseline serum creatinine level was defined as the serum creatinine value on admission or within 6 months before admission. The AKI stage was specified using the AKIN categories as follows: stage 1 (mild)—an increase in the baseline serum creatinine to $\geq 0.3 \mathrm{mg} / \mathrm{dL}$ or $\geq 150-199 \%$; stage 2 (moderate) —an increase in the baseline serum creatinine level to $\geq 200-299 \%$; stage 3 (severe) - an increase in baseline serum creatinine level to $\geq 300 \%$ or the initiation of renal replacement therapy.

Septic shock was defined using the 2016 Third International Consensus Definition for Sepsis and Septic Shock [14], and acute respiratory distress syndrome (ARDS) was defined using the Berlin Definition [15].

\subsection{Clinical Management}

All patients received supportive care, including oxygen therapy. Hospitalized COVID-19 patients were treated with (1) lopinavir/ritonavir or darunavir/cobicistat and (2) with or without hydroxychloroquine. Patients with critical COVID-19 (requiring a reservoir bag-mask oxygen supply/high-flow nasal cannula oxygen supply/mechanical ventilation or shock state) were administered with additional corticosteroid and/or intravenous immunoglobulin based on the physicians' decision. The decision to perform renal replacement therapy was made based on the opinion of the nephrologist and infectious disease specialists.

\subsection{Statistical Analyses}

Continuous variables are presented as median (interquartile range (IQR)) values; categorical variables are presented as numbers (percentage, \%). Mann-Whitney $U$ tests were used to assess differences in continuous variables between AKI and non-AKI patients. Kruskal-Wallis tests with the post hoc Bonferroni test were used to compare the differences in continuous variables among AKI patients. Pearson chi-square tests or Fisher's exact tests were used for categorical variables, as appropriate. The Kaplan-Meier analysis was used to estimate survival, and the log-rank test was used to analyze statistical significance. Univariate and multivariate Cox proportional hazard regression analyses were performed to evaluate the association between AKI severity and patient death. Variables that were potentially associated with mortality were entered into the multivariate analysis, namely age, sex, and complications [7]. Logistic regression analysis was used to investigate the associated factors for stage $3 \mathrm{AKI}$; the selected variables were those previously identified as risk factors for AKI [16,17]. Multivariate logistic regression analysis was performed in a stepwise manner. Variables with an alpha level of risk factor less than 0.10 in the univariate analyses were included; age and sex were included 
regardless of the alpha error. SPSS Statistics for Windows, version 22 (IBM Corp., Armonk, NY, USA) was used for all statistical analyses. A value of $p<0.05$ was considered statistically significant.

\section{Results}

\subsection{Baseline Characteristics}

During the study period, 164 COVID-19 patients were hospitalized in two university-based hospitals; 34 patients matched the AKI definition (Figure S1). Of these, 4 were excluded because they had underlying CKD, and finally, 30 patients (18.3\%) were classified into the AKI group and the remaining 130 patients were classified into the non-AKI group. Among the AKI group, 14 patients $(46.7 \%)$ had stage 1 AKI, $4(13.3 \%)$ had stage 2 AKI, and $12(40.0 \%)$ had stage 3 AKI. The baseline clinical characteristics of the patients are presented in Table 1 . The median age was significantly higher in the AKI group compared with the non-AKI group (75.0 vs. 67.0 years, $p=0.005$ ); the male ratio was $66.7 \%$ in the AKI group and $50.8 \%$ in the non-AKI group. In the AKI group, most patients (28/30, 93.3\%) had at least one comorbid disease, and the rate was significantly higher than those without AKI $(93.3 \%$ vs. $73.8 \%, p=0.02)$, and especially, comorbid diabetes was more common in AKI patients $(46.7 \%$ vs. $27.7 \%, p=0.04)$. There were 17 in-hospital deaths in the AKI group (56.7\%) and 27 deaths in the non-AKI group $(p<0.001)$. In particular, all patients with stage 3 AKI died, and mortality was significantly higher in stage 3 patients than in stage 1 and stage 2 patients (stage 1: 100.0\% vs. 28.6\%, $p<0.001$; stage $2: 100.0 \%$ vs. $25.0 \%, p=0.007$ ).

Among the AKI patients, patients commonly presented with fever $(60.0 \%)$, chills $(53.3 \%)$, fatigue $(66.7 \%)$, cough $(53.3 \%)$, sputum $(46.7 \%)$, and dyspnea $(56.7 \%)$; there was no difference in the signs and symptoms of patients in the different AKI groups. The median duration from symptom onset to admission was 6.5 days and that from symptom onset to AKI diagnosis was 10.0 days. The duration from symptom onset to diagnosis was longer in patients with stage $3 \mathrm{AKI}$ than in those with stage 1 AKI $(p=0.007)$. Vital signs, such as blood pressure and body temperature, were similar among the groups. 
Table 1. Clinical characteristics.

\begin{tabular}{|c|c|c|c|c|c|}
\hline Characteristics & $\begin{array}{l}\text { Non-AKI } \\
(n=130)\end{array}$ & $\begin{array}{l}\text { All AKI } \\
(n=30)\end{array}$ & $\begin{array}{c}\text { AKI Stage } 1 \\
(n=14)\end{array}$ & $\begin{array}{c}\text { AKI Stage } 2 \\
(n=4)\end{array}$ & $\begin{array}{c}\text { AKI Stage } 3 \\
(n=12)\end{array}$ \\
\hline Median age (range), y & $67.0(24.0-92.0)$ & $75.0(60.0-98.0) *$ & $75.0(60.0-98.0)^{*}$ & $73.0(63.0-86.0)$ & $77.0(61.0-87.0)$ \\
\hline Sex, male, $n(\%)$ & $66(50.8)$ & $20(66.7)$ & $8(57.1)$ & $3(75.0)$ & $9(75.0)$ \\
\hline Body mass index, $\mathrm{kg} / \mathrm{m}^{2}$ & $23.4(21.0-26.3)$ & $24.5(22.1-27.1)$ & $25.6(22.8-29.4)$ & $24.0(22.1-26.0)$ & $23.4(20.0-27.1)$ \\
\hline \multicolumn{6}{|l|}{ Comorbid conditions, $n(\%)$} \\
\hline Any comorbidity & $96(73.8)$ & $28(93.3) *$ & $14(100.0) *$ & $3(75.0)$ & $11(91.7)$ \\
\hline Hypertension & $59(45.4)$ & $18(60.0)$ & $11(78.6)^{*}$ & $2(50.0)$ & $5(41.7)$ \\
\hline Diabetes & $36(27.7)$ & $14(46.7) *$ & $7(50.0)$ & $1(25.0)$ & $6(50.0)$ \\
\hline Cardiovascular disease & $16(12.3)$ & $5(16.7)$ & $2(14.3)$ & 0 & $3(25.0)$ \\
\hline Congestive heart failure & $8(6.2)$ & $2(6.7)$ & 0 & 0 & $2(16.7)$ \\
\hline Chronic lung disease & $12(9.2)$ & $4(13.3)$ & 0 & $1(25.0)$ & $3(25.0)$ \\
\hline Cognitive disorder & $26(20.0)$ & $4(13.3)$ & $3(21.4)$ & 0 & $1(8.3)$ \\
\hline Malignancy & $19(14.6)$ & $7(23.3)$ & $2(14.3)$ & $1(25.0)$ & $4(33.3)$ \\
\hline \multicolumn{6}{|l|}{ Signs and symptoms, $n(\%)$} \\
\hline Fever & $93(71.5)$ & $18(60.0)$ & $9(64.3)$ & $3(75.0)$ & $6(50.0)$ \\
\hline Chill & $60(46.2)$ & $16(53.3)$ & $9(64.3)$ & $1(25.0)$ & $6(50.0)$ \\
\hline Myalgia & $40(30 ., 8)$ & $9(30.0)$ & $5(35.7)$ & 0 & $4(33.3)$ \\
\hline Fatigue & $63(48.5)$ & $20(66.7)$ & $10(71.4)$ & $2(50.0)$ & $8(66.7)$ \\
\hline Cough & $45(34.6)$ & $16(53.3)$ & $7(50.0)$ & $1(25.0)$ & $8(66.7)$ \\
\hline Sputum & $35(26.9)$ & $14(46.7) *$ & $6(42.9)$ & $1(25.0)$ & $7(58.3) *$ \\
\hline Dyspnea & $58(44.6)$ & $17(56.7)$ & $8(57.1)$ & $2(50.0)$ & $7(58.3)$ \\
\hline Rhinorrhea & $10(7.7)$ & $2(6.7)$ & 0 & $1(25.0)$ & $1(8.3)$ \\
\hline Nausea or vomiting & $10(7.7)$ & $2(6.7)$ & 0 & $1(25.0)$ & $1(8.3)$ \\
\hline Diarrhea & $19(14.6)$ & $3(10.0)$ & $2(14.3)$ & $1(25.0)$ & 0 \\
\hline Days from symptom onset to diagnosis & $5.0(3.0-10.0)$ & $4.0(3.0-7.5)$ & $3.5(1.0-6.3)$ & $4.5(1.0-6.5)$ & $5.5(3.5-10.5)$ \\
\hline Days from symptom onset to admission & $6.0(3.0-10.0)$ & $6.5(4.0-12.0)$ & $5.5(3.0-10.0)$ & $7.5(4.5-12.0)$ & $8.5(5.0-15.0)$ \\
\hline Days from symptom onset to diagnosis of AKI & & $10.0(5.8-19.0)$ & $8.0(4.0-13.0)^{\mathrm{a}}$ & $8.5(5.0-27.8)^{a, b}$ & $17.5(9.8-25.0)^{b}$ \\
\hline Length of hospital stay, days & $16.0(9.0-25.0)$ & $20.0(12.0-31.0)$ & $23.5(14.0-36.0)$ & $34.0(10.5-57.5)$ & $17.0(8.8-24.5)$ \\
\hline In-hospital death & $27(20.8)$ & $17(56.7) *$ & $4(28.6)^{a}$ & $1(25.0)^{b}$ & $12(100.0)^{*}, \mathrm{c}$ \\
\hline \multicolumn{6}{|l|}{ Vital signs } \\
\hline Systolic BP, mmHg & $133.5(116.5-148.0)$ & $134.0(109.8-155.5)$ & $115.0(106.0-145.5)$ & $134.5(128.0-155.3)$ & $150.0(115.5-169.3)$ \\
\hline Diastolic BP, mmHg & $78.0(68.0-86.5)$ & $71.0(60.0-91.3)$ & $66.5(59.3-84.5)$ & $79.5(70.5-90.0)$ & $73.5(59.0-98.0)$ \\
\hline
\end{tabular}


Table 1. Cont.

\begin{tabular}{|c|c|c|c|c|c|}
\hline Characteristics & $\begin{array}{l}\text { Non-AKI } \\
(n=130)\end{array}$ & $\begin{array}{l}\text { All AKI } \\
(n=30)\end{array}$ & $\begin{array}{c}\text { AKI Stage } 1 \\
(n=14)\end{array}$ & $\begin{array}{c}\text { AKI Stage } 2 \\
(n=4)\end{array}$ & $\begin{array}{c}\text { AKI Stage } 3 \\
(n=12)\end{array}$ \\
\hline Heart rate, beats/min & $88.0(79.0-102.0)$ & $86.0(79.0-108.0)$ & $79.5(76.5-95.3)$ & $86.5(83.5-103.0)$ & $91.5(80.0-109.5)$ \\
\hline Body temperature, ${ }^{\circ} \mathrm{C}$ & $36.9(36.4-37.5)$ & $37.3(36.8-37.9)$ & $37.2(36.7-38.0)$ & $37.4(37.0-37.7)$ & $37.2(36.5-38.0)$ \\
\hline \multicolumn{6}{|c|}{ Chest radiography findings, $n(\%)$} \\
\hline Patchy consolidation & $73(56.2)$ & $16(53.3)$ & $9(64.3)$ & $1(25.0)$ & $6(50.0)$ \\
\hline Ground glass opacity & $64(49.2)$ & $15(50.0)$ & $6(42.9)$ & $2(50.0)$ & $7(58.3)$ \\
\hline No active lung lesion & $24(18.5)$ & $4(13.3)$ & $2(14.3)$ & $1(25.0)$ & $1(8.3)$ \\
\hline
\end{tabular}




\subsection{Laboratory Findings on Admission among AKI Patients}

The laboratory indices on admission among AKI patients are presented in Table 2. The median serum albumin level was $3.3 \mathrm{mg} / \mathrm{dL}$; the level was lower in patients with stage $3 \mathrm{AKI}$ than in those with stage $1 \mathrm{AKI}(p=0.01)$. In renal function categories, the median serum blood urea nitrogen, creatinine, and eGFR were $23.7 \mathrm{mg} / \mathrm{dL}, 1.1 \mathrm{mg} / \mathrm{dL}$, and $60.0 \mathrm{~mL} / \mathrm{min} / 1.73 \mathrm{~m}^{2}$, respectively; they were not different between the groups.

Table 2. Laboratory findings on admission in AKI patients.

\begin{tabular}{|c|c|c|c|c|}
\hline Laboratory Findings & $\begin{array}{l}\text { All AKI } \\
(n=30)\end{array}$ & $\begin{array}{l}\text { AKI Stage } 1 \\
\quad(n=14)\end{array}$ & $\begin{array}{c}\text { AKI Stage } 2 \\
\quad(n=4)\end{array}$ & $\begin{array}{c}\text { AKI Stage } 3 \\
\quad(n=12)\end{array}$ \\
\hline White blood cell count, $\times 10^{9} / \mathrm{L}$ & $7.1(5.8-11.9)$ & $7.1(5.5-10.1)$ & $6.0(4.4-8.2)$ & $9.9(6.7-14.4)$ \\
\hline Absolute neutrophil count, $\times 10^{9} / \mathrm{L}$ & $6.3(4.0-10.8)$ & $5.9(3.9-8.8)$ & $4.9(3.6-7.1)$ & $9.5(5.6-14.2)$ \\
\hline Lymphocyte count, $\times 10^{9} / \mathrm{L}$ & $0.7(0.5-0.9)$ & $0.9(0.7-1.1)$ & $0.6(0.5-0.7)$ & $0.6(0.5-0.9)$ \\
\hline Monocyte count, $\times 10^{9} / \mathrm{L}$ & $0.3(0.2-0.4)$ & $0.3(0.3-0.4)$ & $0.2(0.2-0.3)$ & $0.3(0.1-0.6)$ \\
\hline Hemoglobin, g/dL & $12.3(10.9-13.9)$ & $12.3(10.9-13.2)$ & $13.3(10.8-14.7)$ & $12.2(10.6-13.8)$ \\
\hline Platelet count, $\times 10^{9} / \mathrm{L}$ & $241.0(179.5-296.8)$ & $255.5(208.0-296.0)$ & $204.5(170.0-351.8)$ & $214.0(140.5-302.8)$ \\
\hline hs-CRP, mg/dL & $10.3(6.1-20.9)$ & $6.8(4.9-16.3)$ & $14.4(5.8-24.4)$ & $17.4(8.6-26.0)$ \\
\hline Procalcitonin, ng/mL $(n=16)$ & $0.18(0.09-0.70)$ & $0.03(0.02-0.19)$ & $0.10(0.09-0.16)$ & $0.22(0.15-0.91)$ \\
\hline Ferritin, ng/mL $(n=19)$ & $531.0(255.0-868.0)$ & $483.7(193.3-657.6)$ & $868.0(484.0-920.0)$ & $520.8(309.0-7450.0)$ \\
\hline AST, U/L & $48.0(36.0-63.3)$ & $47.0(34.5-69.3)$ & $48.5(38.8-50.8)$ & $50.0(33.0-70.5)$ \\
\hline ALT, U/L & $26.0(17.8-34.5)$ & $27.5(16.3-38.3)$ & $19.5(19.0-24.5)$ & $27.5(20.3-35.5)$ \\
\hline Total bilirubin, mg/dL & $0.6(0.4-0.9)$ & $0.5(0.4-0.8)$ & $1.1(0.6-1.4)$ & $0.7(0.5-0.9)$ \\
\hline Total protein, g/dL & $6.7(6.4-7.1)$ & $6.9(6.6-7.4)$ & $6.6(6.4-6.9)$ & $6.5(6.1-7.0)$ \\
\hline Albumin, g/dL & $3.3(3.0-3.5)$ & $3.5(3.2-3.7)^{a}$ & $3.5(3.2-3.8)^{a, b}$ & $3.2(2.7-3.3)^{b}$ \\
\hline BUN, mg/dL & $23.7(15.7-32.0)$ & $23.7(18.7-39.3)$ & $23.3(12.1-25.1)$ & $23.8(14.6-41.5)$ \\
\hline Creatinine, mg/dL & $1.1(0.8-1.6)$ & $1.1(1.0-1.4)$ & $1.1(0.7-1.6)$ & $1.1(0.7-1.9)$ \\
\hline $\mathrm{eGFR}, \mathrm{mL} / \mathrm{min} / 1.73 \mathrm{~m}^{2}$ & $60.0(41.5-82.0)$ & $53.5(41.5-69.8)$ & $67.0(33.8-97.3)$ & $61.5(38.0-86.5)$ \\
\hline LDH, U/L $(n=25)$ & $481.0(286.0-593.0)$ & $481.0(249.0-589.5)$ & $475.5(277.0-475.5)$ & $492.5(306.0-592.5)$ \\
\hline $\mathrm{CPK}, \mathrm{U} / \mathrm{L}(n=17)$ & $135.0(72.5-533.5)$ & $102.0(63.5-436.8)$ & $220.0(68.0-220.0)$ & $494.0(177.0-837.5)$ \\
\hline D-dimer, $\mu \mathrm{g} / \mathrm{mL}(n=16)$ & $2.1(0.8-3.2)$ & $2.1(1.0-2.6)$ & $1.2(0.2-1.2)$ & $2.0(0.8-6.9)$ \\
\hline Sodium, $\mathrm{mEq} / \mathrm{L}$ & $135.5(133.0-139.3)$ & $137.0(132.8-142.0)$ & $135.0(132.5-137.5)$ & $133.5(133.0-138.5)$ \\
\hline Potassium, $\mathrm{mEq} / \mathrm{L}$ & $4.0(3.3-4.7)$ & $3.8(3.5-4.9)^{\mathrm{a}}$ & $3.2(3.1-3.3)^{b}$ & $4.4(3.6-5.2)^{a, b}$ \\
\hline Lactate, mEq/L & $1.9(1.4-2.5)$ & $1.9(1.5-2.5)$ & $1.1(1.1-1.9)$ & $2.0(1.4-3.2)$ \\
\hline Prothrombin time, $\mathrm{s}$ & $12.4(11.9-13.0)$ & $12.2(11.7-12.6)$ & $12.6(12.2-13.0)$ & $12.1(11.8-15.1)$ \\
\hline Activated partial thromboplastin time, s & $31.4(28.7-33.5)$ & $31.2(26.1-34.4)$ & $30.0(28.6-31.2)$ & $32.2(30.9-34.3)$ \\
\hline
\end{tabular}

The different superscripts $(a, b)$ denote significant differences between groups not sharing the same superscript at the 0.05 level. Data are expressed as median (IQR) or $n(\%)$. Abbreviations: AKI, acute kidney injury; hs-CRP, high-sensitivity C-reactive protein; AST, aspartate aminotransferase; ALT, alanine aminotransferase; BUN, blood urea nitrogen; eGFR, estimated glomerular filtration rate; $\mathrm{LDH}$, lactate dehydrogenase; $\mathrm{CPK}$, creatine phosphokinase.

\subsection{Treatment and Complications among AKI Patients}

The in-hospital treatments and complications among AKI patients are summarized in Table 3. About $96.7 \%$ of AKI patients were treated with lopinavir/ritonavir or darunavir/cobicistat, and $83.3 \%$ were treated with hydroxychloroquine. There was no difference in the duration of the use of these drugs among AKI groups. Stage 3 AKI patients were given inotropic agents more commonly than stage 1 AKI patients $(p=0.01)$. Stage 3 AKI patients had a higher incidence of ARDS than stage 1 AKI patients $(p=0.01)$. 
Table 3. Treatment and complications in AKI patients.

\begin{tabular}{ccccc}
\hline Variables & $\begin{array}{c}\text { All AKI } \\
(\boldsymbol{n}=\mathbf{3 0})\end{array}$ & $\begin{array}{c}\text { AKI Stage 1 } \\
(\boldsymbol{n}=\mathbf{1 4})\end{array}$ & $\begin{array}{c}\text { AKI Stage 2 } \\
(\boldsymbol{n}=\mathbf{4})\end{array}$ & $\begin{array}{c}\text { AKI Stage 3 } \\
(\boldsymbol{n}=\mathbf{1 2})\end{array}$ \\
\hline Treatments, $\boldsymbol{n}(\mathbf{\%})$ & & & & \\
Lopinavir/ritonavir or darunavir/cobicistat & $29(96.7)$ & $14(100.0)$ & $4(100.0)$ & $11(91.7)$ \\
Hydroxychloroquine & $25(83.3)$ & $12(85.7)$ & $4(100.0)$ & $9(75.0)$ \\
Antibiotics & $29(96.7)$ & $13(92.9)$ & $4(100.0)$ & $12(100.0)$ \\
Glucocorticoid & $18(60.0)$ & $6(42.9)$ & $2(50.0)$ & $10(83.3)$ \\
Intravenous immunoglobulin & $5(16.7)$ & $1(7.1)$ & 0 & $4(33.3)$ \\
Inotropic agents & $17(56.7)$ & $5(35.7)^{\mathrm{a}}$ & $2(50.0)^{\mathrm{a}, \mathrm{b}}$ & $10(83.3)^{\mathrm{b}}$ \\
Oxygen therapy & $29(96.7)$ & $13(92.9)$ & $4(100.0)$ & $12(100.0)$ \\
Low-flow oxygen & $5(16.7)$ & $3(21.4)$ & $2(50.0)$ & 0 \\
High-flow oxygen & $8(26.7)$ & $5(35.7)$ & 0 & $3(25.0)$ \\
Invasive mechanical ventilation & $16(53.3)$ & $5(35.7)$ & $2(50.0)$ & $9(75.0)$ \\
Continuous renal replacement therapy & $5(16.7)$ & 0 & 0 & $5(41.7)^{\mathrm{b}}$ \\
Extracorporeal membrane oxygenation & $1(3.3)$ & 0 & 0 & $1(8.3)$ \\
ICU admission & $20(66.7)$ & $8(57.1)$ & $2(50.0)$ & $10(83.3)$ \\
Duration of medication used, days (IQR) & & & & \\
Lopinavir/ritonavir or darunavir/cobicistat & $8.0(5.0-10.0)$ & $7.5(5.0-10.0)$ & $10.5(8.0-12.0)$ & $8.0(4.0-9.5)$ \\
Hydroxychloroquine & $5.0(2.0-10.0)$ & $5.0(2.0-10.0)$ & $6.5(3.0-9.5)$ & $4.0(1.5-9.5)$ \\
Complications, $\boldsymbol{n}$ (\%) & & & & \\
ARDS & $17(56.7)$ & $5(35.7)^{\mathrm{a}}$ & $2(50.0)^{\mathrm{a}, \mathrm{b}}$ & $10(83.3)^{\mathrm{b}}$ \\
Septic shock & $14(46.7)$ & $4(28.6)$ & $1(25.0)$ & $9(75.0)$ \\
Arrhythmia & $2(6.7)$ & $1(7.1)$ & 0 & $1(8.3)$ \\
DIC & $2(6.7)$ & 0 & 0 & $2(16.7)$ \\
\hline
\end{tabular}

The different superscripts $(a, b)$ denote significant differences between groups not sharing the same superscript at the 0.05 level. Abbreviations: AKI, acute kidney injury; ARDS, acute respiratory distress syndrome; DIC, disseminated intravascular coagulation.

\subsection{Severity of Acute Kidney Injury and In-Hospital Mortality}

In the Kaplan-Meier survival curve, 30-day mortality was significantly higher in the stage 3 AKI group than in the non-AKI and the stage 1 and 2 AKI groups (log-rank $p<0.001$, Figure 1 ). In all hospitalized COVID-19 patients, stage 3 AKI and age were independently associated with patient survival after adjusting for confounding factors, such as sex, hypertension, and diabetes (stage 3 AKI: hazard ratio $(\mathrm{HR})=3.62(95 \%$ confidence interval $(\mathrm{CI})=1.75-7.48), p=0.001$; age: $\mathrm{HR}=1.04$ $(95 \%$ CI $=1.01-1.07, p=0.003))($ Table 4$)$.

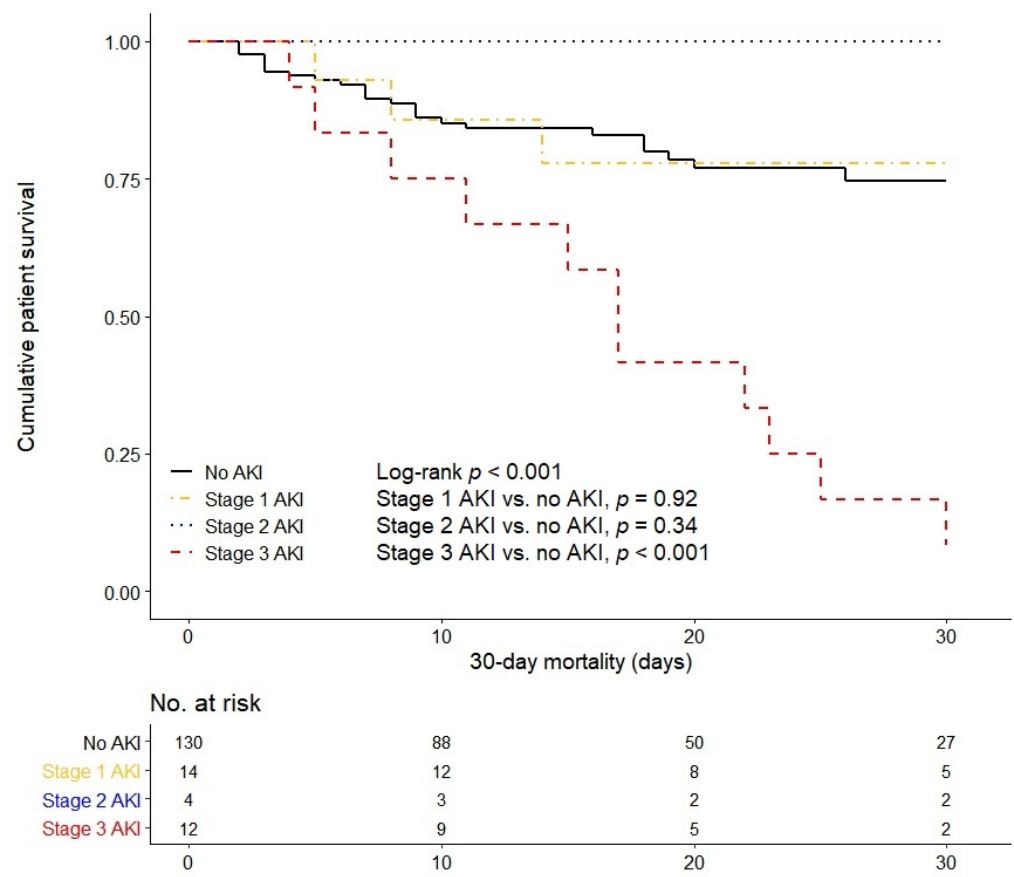

Figure 1. Kaplan-Meier survival curve for 30-day mortality based on the severity of acute kidney injury. 
Table 4. Effect of AKI on mortality among all hospitalized COVID-19 patients.

\begin{tabular}{ccccccc}
\hline \multirow{2}{*}{ Variables } & \multicolumn{2}{c}{ Univariate } & \multicolumn{2}{c}{ Model 1 } & \multicolumn{2}{c}{ Model 2 $\ddagger$} \\
\cline { 2 - 7 } & HR (95\% CI) & $p$ & HR (95\% CI) & $p$ & HR (95\% CI) & $p$ \\
\hline AKIN & & & & & & \\
No AKI & Reference & & Reference & & Reference & \\
Stage 1 & $0.98(0.34-2.81)$ & 0.97 & $0.68(0.23-1.98)$ & 0.48 & $0.59(0.20-1.75)$ & 0.34 \\
Stage 2 & $0.63(0.08-4.76)$ & 0.65 & $0.45(0.06-3.42)$ & 0.45 & $0.42(0.06-3.26)$ & 0.41 \\
Stage 3 & $5.28(2.65-10.55)$ & $<0.001$ & $3.77(1.84-7.71)$ & $<0.001$ & $3.62(1.75-7.48)$ & 0.001 \\
Age & $1.04(1.01-1.06)$ & 0.003 & $1.04(1.01-1.07)$ & 0.003 & $1.04(1.01-1.07)$ & 0.003 \\
Sex (ref: F) & $1.52(0.82-2.80)$ & 0.19 & $0.63(0.33-1.20)$ & 0.16 & $0.61(0.32-1.16)$ & 0.13 \\
Hypertension & $1.44(0.79-2.63)$ & 0.24 & & & $1.34(0.71-2.52)$ & 0.36 \\
Diabetes & $1.55(0.85-2.83)$ & 0.15 & & & $1.35(0.72-2.56)$ & 0.35 \\
\hline
\end{tabular}

${ }^{\dagger}$ Adjusted for age and sex; ${ }^{\ddagger}$ Additionally adjusted for hypertension and diabetes. Abbreviations: AKI, acute kidney injury; COVID-19, coronavirus disease 2019; HR, hazard ratio; CI, confidence interval; AKIN, Acute Kidney Injury Network.

Among the AKI patients, the in-hospital mortality was significantly higher in stage 3 AKI patients than in stage 1 AKI patients $(\mathrm{HR}=7.58(95 \% \mathrm{CI}=2.04-28.24), p=0.003)$ (Table 5$)$. The association between stage $3 \mathrm{AKI}$ and higher in-hospital mortality remained significant after adjusting for confounding factors: age and sex (model 1: $\mathrm{HR}=7.66(95 \% \mathrm{CI}=2.03-28.86), p=0.003)$; age, sex, ARDS, and septic shock (model 2: HR $=15.65(95 \% \mathrm{CI}=2.43-100.64), p=0.004)$. In model 2 , ARDS and septic shock also had an association with in-hospital mortality (ARDS: $\mathrm{HR}=12.24(95 \% \mathrm{CI}=1.87-79.92), p=0.01$; septic shock: $\mathrm{HR}=11.42(95 \% \mathrm{CI}=1.59-82.25), p=0.02)$.

Table 5. Effect of AKI on mortality among AKI patients.

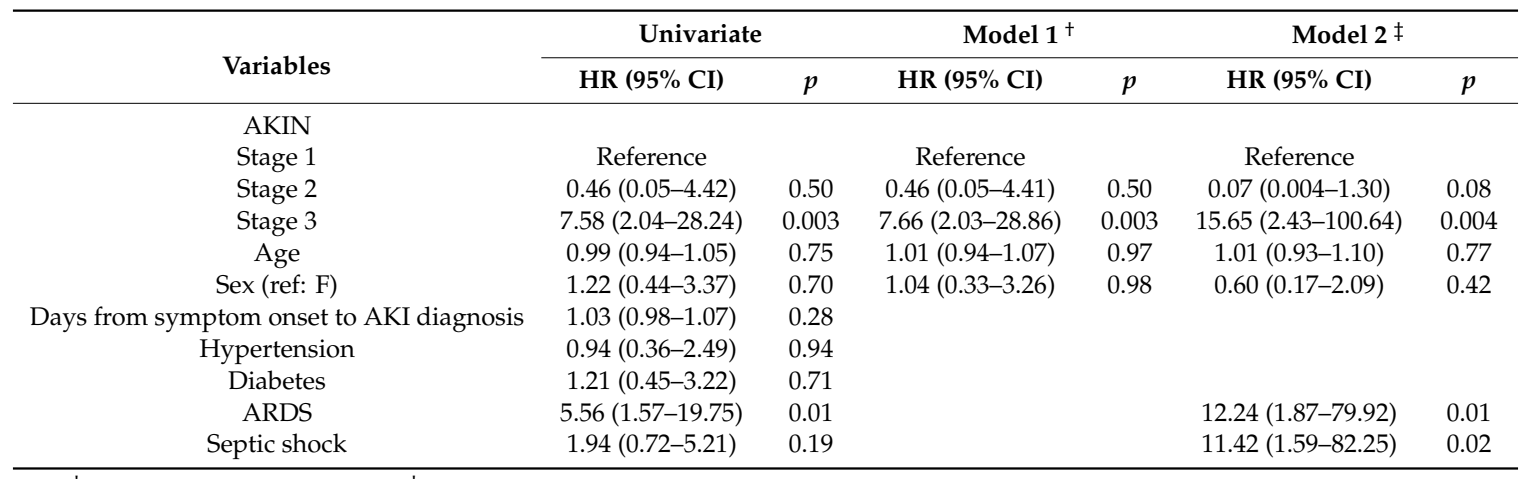

${ }^{\dagger}$ Adjusted for age and sex; ${ }^{\ddagger}$ Additionally adjusted for ARDS and septic shock. Abbreviations: AKI, acute kidney injury; HR, hazard ratio; CI, confidence interval; AKIN, Acute Kidney Injury Network; ARDS, acute respiratory distress syndrome.

\subsection{Predictors for Severe Acute Kidney Injury}

In the univariate logistic regression analysis, the number of days from symptom onset to diagnosis of AKI, ARDS, septic shock, and serum albumin level on admission were associated with stage 3 AKI (all $p<0.05$ ) (Table 6). In the multivariate stepwise logistic regression analysis adjusted for age and sex, ARDS and serum albumin on admission were independent predictors for stage 3 AKI (ARDS: odds ratio $(\mathrm{OR})=9.05(95 \% \mathrm{CI}=1.05-78.14), p=0.04$; serum albumin: $\mathrm{OR}=0.02(95 \% \mathrm{CI}=0.001-0.87)$, $p=0.04)$. 
Table 6. Risk factors for severe AKI among AKI patients in the logistic regression analysis.

\begin{tabular}{ccccc}
\hline \multirow{2}{*}{ Variables } & Univariate & \multicolumn{2}{c}{ Multivariate $^{+}$} \\
\cline { 2 - 5 } & OR (95\% CI) & $p$ & OR (95\% CI) & $p$ \\
\hline Age & $0.98(0.90-1.07)$ & 0.65 & $1.01(0.87-1.16)$ & 0.91 \\
Sex (ref: F) & $1.91(0.38-9.59)$ & 0.43 & $1.17(0.15-9.09)$ & 0.88 \\
Days from symptom onset to AKI diagnosis & $1.11(1.00-1.23)$ & 0.04 & & \\
Hypertension & $0.28(0.06-1.29)$ & 0.10 & & 0.04 \\
Diabetes & $1.25(0.29-5.41)$ & 0.77 & & \\
ARDS & $7.86(1.31-47.04)$ & 0.02 & $9.05(1.05-78.14)$ & \\
Septic shock & $7.80(1.48-41.21)$ & 0.02 & & 0.04 \\
\hline Serum albumin on admission & $0.05(0.004-0.69)$ & 0.03 & $0.02(0.001-0.87)$ & \\
\hline
\end{tabular}

${ }^{\dagger}$ Variables with the alpha level of risk factor less than 0.10 in univariate analyses were included in the logistic regression model with backward selection; age and gender were included in the model regardless of the alpha level. Abbreviations: AKI, acute kidney injury; OR, odds ratio; CI, confidence interval; ARDS, acute respiratory distress syndrome.

\subsection{Renal Replacement Therapy}

Five out of 30 (16.7\%) AKI patients underwent dialysis, all of whom were treated with continuous renal replacement therapy (CRRT) (Table 7). There were various indications for dialysis, which were as follows: hyperkalemia (2/5), hypervolemia (2/5), and hypervolemia/metabolic acidosis (1/5). The serum creatinine levels varied between 1.29 and $5.03 \mathrm{mg} / \mathrm{dL}$. Despite dialysis, all five patients eventually died. 
Table 7. Information regarding patients who underwent continuous renal replacement therapy.

\begin{tabular}{|c|c|c|c|c|c|c|c|c|c|c|c|c|c|}
\hline Patient No. & Age & Sex & SAPS & SOFA & APACHEII & Indication & $\begin{array}{c}\text { Screat on CRRT } \\
\text { Initiation } \\
\text { (mg/dL) }\end{array}$ & $\begin{array}{l}\text { Admission to } \\
\text { CRRT Initiation } \\
\text { (days) }\end{array}$ & Anticoagulation & $\begin{array}{l}\text { Duration } \\
\text { (hrs) }\end{array}$ & $\begin{array}{c}\text { Effluent } \\
\text { Flow Rate } \\
(\mathrm{mL} / \mathrm{kg} / \mathrm{h})\end{array}$ & Outcome & $\begin{array}{l}\text { Change } \\
\text { to HD }\end{array}$ \\
\hline 1 & 67 & $\mathrm{M}$ & 72 & 12 & 28 & Hyperkalemia & 1.29 & 6 & Heparin & 155 & 11 & Death & No \\
\hline 2 & 75 & $\mathrm{~F}$ & 83 & 19 & 34 & Hypervolemia & 5.03 & 9 & Heparin & 560 & 33 & Death & No \\
\hline 3 & 60 & M & 79 & 15 & 30 & Hypervolemia and acidosis & 3.47 & 6 & Heparin & 237 & 28 & Death & No \\
\hline 4 & 67 & $\mathrm{~F}$ & 57 & 10 & 19 & Hypervolemia & 2.82 & 2 & Nafamostat & 26 & 29 & Death & No \\
\hline 5 & 78 & $\mathrm{M}$ & 58 & 10 & 23 & Hyperkalemia & 2.90 & 7 & Nafamostat & 21 & 25 & Death & No \\
\hline
\end{tabular}

Abbreviations: SAPS, Simplified Acute Physiology Score; SOFA, Sequential Organ Failure Assessment; APACHE, Acute Physiology and Chronic Health Evaluation; Screat, serum creatinine; CRRT, continuous renal replacement therapy; HD, hemodialysis. 


\section{Discussion}

This Korean COVID-19 study demonstrated that COVID-19 patients with AKI were not rare among hospitalized COVID-19 patients with severe to critical symptoms. The overall mortality of these patients was high; in particular, patients with severe AKI showed fatal outcomes and severe AKI was found to be an independent predictor of in-hospital death. Thus, clinicians should pay special attention to the treatment of COVID-19 patients with severe AKI.

Recently reported COVID-19 studies have found a prevalence rate of $0-15 \%$ for patients with AKI complicating COVID-19 $[7,18,19]$. The wide range of prevalence may be mainly caused by the differences in the disease severity. In this study, after excluding patients with pre-existing CKD, $18.3 \%$ of patients showed a complication with AKI among hospitalized COVID-19 patients. Based on their analyses of 116 COVID-19 patients, Wang et al. reported no AKI cases, except for those who had CKD [18]. However, patients in that study were relatively young and had mild symptoms such that the mortality in that study was only $6.0 \%$ (7/116). In contrast, the mortality of total hospitalized patients was $26.8 \%$ in our study (44/164). This difference seems to be partly associated with the characteristics of our patients. South Korea has allocated patients into professional COVID-19 treatment hospitals, community hospitals, and therapeutic living centers according to the severity score of COVID-19, which was based on age, underlying diseases (hypertension, diabetes, end-stage renal disease, congestive heart failure, chronic lung disease, and malignancy), and social factors (living in long-term care facilities and facilities for the disabled) [11]. As our hospital was designated as a professional COVID-19 treatment center, patients were screened and only those who showed a higher severity of COVID-19 were hospitalized. Therefore, patients with mild to moderate symptoms were excluded in this analysis, even if they were elderly. According to our results, the AKI incidence rate among severe or critical COVID-19 patients was up to $18 \%$. In patients with severe to critical COVID-19, the risk of complicating AKI should be considered and frequent renal function monitoring is recommended.

Among hospitalized COVID-19 patients with severe to critical symptoms, patients who developed AKI were older and had comorbid diabetes compared with those without AKI. Previous studies have also identified these as risk factors for AKI in hospitalized and critically ill viral infection patients [20,21]. Additionally, a recent multicenter study in New York has found older age and diabetes as independent risk factors for AKI in COVID-19 patients [22]. According to our results, elderly COVID-19 patients who had diabetes need to be closely monitored for the development of AKI, and the prognosis of COVID-19 will be fatal in severe AKI patients, especially in the elderly. However, the prognosis of patients with mild to moderate AKI may not be serious.

$\mathrm{AKI}$ is common in hospitalized patients and is a risk factor for mortality [23]; the mortality rate is almost 70\% in critically ill patients with septic AKI [24]. Stage 3 AKI patients have 6.8 times higher mortality than those without AKI among hospitalized patients [25]. Recently, Cheng et al. reported higher mortality in COVID-19 AKI patients [7]. A total of 36 out of 701 patients (5.1\%) developed AKI, but among them, 14 already had CKD. After adjusting for confounding factors, stage 2 and stage 3 AKI patients were found to have 3.5 times and 4.7 times higher mortality than those with normal kidney function, respectively. However, in our study, mild and moderate AKI patients did not differ in terms of mortality; severe AKI patients had 3.6 times higher risk of in-hospital death than non-AKI patients and also had higher mortality compared with mild and moderate AKI patients. This difference might be attributable to the difference in patient characteristics and treatment. We excluded patients with CKD because the degree of AKI and the effect of AKI on prognosis could not be accurately evaluated in these patients. Moreover, many of our patients were treated with lopinavir/ritonavir or darunavir/cobicistat $(29 / 30,96.7 \%)$ and hydroxychloroquine (25/30, 83.3\%); however, Cheng et al. reported that only $28 \%$ of the patients were given lopinavir/ritonavir without hydroxychloroquine, a potential therapeutic agent against COVID-19 [26]. A well-designed randomized controlled trial on a large patient population is required to confirm the effect of treatment on mortality. 
Regarding the characteristics of AKI patients, those with severe AKI had a longer duration from symptom onset to AKI diagnosis and a worse level of inflammatory markers, such as albumin, compared with those with mild AKI. According to the results, patients with long periods from symptom onset to hospitalization, those with a longer hospitalization period, or those with severe infection signs on admission would require frequent check-ups for renal function; in particular, those with ARDS or low albumin on admission were at high risk for severe AKI.

Most AKI COVID-19 patients $(28 / 30,93.3 \%)$ had underlying diseases, though one severe AKI patient had no underlying disease (1/12,8.3\%; patient number 4 in Table 7$)$. The initial serum creatinine level of this patient was normal; however, AKI occurred 1 day after admission and CRRT was initiated. Nonetheless, the disease advanced rapidly and the patient died on day 4 of hospitalization. Therefore, even if there is no underlying disease, COVID-19 patients with severe AKI have a high risk of mortality, warranting extra care from the treating doctors.

The detailed pathophysiologic relationship between COVID-19 and AKI remains unclear. However, the role of angiotensin-converting enzyme 2 (ACE2) is emerging. ACE2 has been identified as the receptor for SARS-CoV-1, and was recently confirmed as a cell entry receptor for SARS-CoV-2, potentially affecting the infectiousness of both SARS viruses $[27,28]$. ACE2s are expressed in many organs, especially the gastrointestinal organs and the kidney. In human tissue RNA sequencing, ACE2 expression is nearly 100 times higher in the kidneys than in the lungs [29]; thus, kidneys with abundant ACE2 expressions can be the main target of SARS-CoV-2 infection. Recent studies that have evaluated the renal histopathologic findings in COVID-19 support the direct renal infection of SARS-CoV-2 [30,31]. Additionally, a viral infection produces inflammatory mediators and can cause a cytokine storm; this results in the downstream cascade of signals and increased synthesis of proinflammatory cytokines and oxidative stress [32]. Thus, endothelial dysfunction and microvascular injury occur and cause AKI. Moreover, hypoperfusion caused by severe infection and the use of nephrotoxic agents, such as antibiotics, may contribute to AKI $[9,33]$.

This study has certain limitations. First, the number of patients with AKI was small and the follow-up period was short; therefore, there is a possibility of type II errors. Furthermore, there was no information on the progress after discharge. Second, the Ct values of rRT-PCR could not be verified in all patients as primary screening centers often confirmed COVID-19 and transferred the confirmed patients with severe to critical symptoms to our hospitals. Third, we could not identify the effect of azithromycin that showed a reinforcing effect for hydroxychloroquine in a recent study [34]. Nonetheless, to our knowledge, this is the first study to demonstrate the risk of COVID-19 that was complicated with severe AKI in patients outside of China.

\section{Conclusions}

COVID-19 patients with severe AKI had fatal outcomes; however, the mortality rate was not increased in patients with mild and moderate AKI. Among AKI patients, those with ARDS or a low serum albumin level on admission had a higher incidence of severe AKI. Clinicians need to pay more attention to prevent and manage severe AKI in COVID-19 patients.

Supplementary Materials: The following are available online at http://www.mdpi.com/2077-0383/9/6/1718/s1, Figure S1: Flow diagram of the study participants.

Author Contributions: Conceptualization, J.-H.L., J.-H.C., and Y.-L.K.; data curation, J.-H.L. and Y.J.; formal analysis, J.-H.L. and Y.J.; funding acquisition, S.-H.P.; investigation, J.-H.C., H.-Y.J., J.-Y.C., C.-D.K., Y.-H.L., H.S., J.L., K.T.K., S.-W.K., and H.-H.C.; methodology, J.-H.L. and S.-H.P.; project administration, Y.-L.K.; supervision, H.-H.C. and Y.-L.K.; writing-original draft, J.-H.L. and S.-H.P.; writing-review and editing, H.-H.C. and Y.-L.K. All authors have read and agreed to the published version of the manuscript.

Acknowledgments: We would like to thank all medical staff for their effort in COVID-19 patient care. This study was supported by research grants from the Daegu Medical Association COVID-19 scientific committee and the Korea Health Technology R\&D Project through the Korea Health Industry Development Institute (KHIDI), funded by the Ministry of Health \& Welfare, Republic of Korea (grant number: HI15C0001).

Conflicts of Interest: All authors declare no competing interests. 


\section{References}

1. Guan, W.-J.; Ni, Z.-Y.; Hu, Y.; Liang, W.-H.; Ou, C.-Q.; He, J.-X.; Liu, L.; Shan, H.; Lei, C.-L.; Hui, D.S.C.; et al. Clinical Characteristics of Coronavirus Disease 2019 in China. N. Engl. J. Med. 2020. [CrossRef] [PubMed]

2. Huang, C.; Wang, Y.; Li, X.; Ren, L.; Zhao, J.; Hu, Y.; Zhang, L.; Fan, G.; Xu, J.; Gu, X.; et al. Clinical features of patients infected with 2019 novel coronavirus in Wuhan, China. Lancet 2020, 395, 497-506. [CrossRef]

3. Zhu, N.; Zhang, D.; Wang, W.; Li, X.; Yang, B.; Song, J.; Zhao, X.; Huang, B.; Shi, W.; Lu, R.; et al. A Novel Coronavirus from Patients with Pneumonia in China, 2019. N. Engl. J. Med. 2020, 382, 727-733. [CrossRef]

4. World Health Organization. Novel Coronavirus (2019-nCoV) Situation Report-51. 2020. Available online: https://www.who.int/docs/default-source/coronaviruse/situation-reports/20200311-sitrep51-covid-19.pdf? (accessed on 26 April 2020).

5. Boldog, P.; Tekeli, T.; Vizi, Z.; Dénes, A.; Bartha, F.A.; Röst, G. Risk Assessment of Novel Coronavirus COVID-19 Outbreaks Outside China. J. Clin. Med. 2020, 9, 571. [CrossRef] [PubMed]

6. Naicker, S.; Yang, C.W.; Hwang, S.J.; Liu, B.C.; Chen, J.H.; Jha, V. The Novel Coronavirus 2019 epidemic and kidneys. Kidney Int. 2020. [CrossRef]

7. Cheng, Y.; Luo, R.; Wang, K.; Zhang, M.; Wang, Z.; Dong, L.; Li, J.; Yao, Y.; Ge, S.; Xu, G. Kidney disease is associated with in-hospital death of patients with COVID-19. Kidney Int. 2020. [CrossRef]

8. Yin, Y.; Wunderink, R.G. MERS, SARS and other coronaviruses as causes of pneumonia. Respirology 2018, 23, 130-137. [CrossRef]

9. Chu, K.H.; Tsang, W.K.; Tang, C.S.; Lam, M.F.; Lai, F.M.; To, K.F.; Fung, K.S.; Tang, H.L.; Yan, W.W.; Chan, H.W.; et al. Acute renal impairment in coronavirus-associated severe acute respiratory syndrome. Kidney Int. 2005, 67, 698-705. [CrossRef]

10. Alkindi, F.; Boobes, Y.; Chandrasekhar Nair, S.; Hashmey, R. SAT-028 acute kidney injury associated with middle east respiratory syndrome coronavirus (MERS-CoV) infection. Kidney Int. Rep. 2020, 5, S13. [CrossRef]

11. Kim, S.-W.; Lee, K.S.; Kim, K.; Lee, J.J.; Kim, J.-Y. A Brief Telephone Severity Scoring System and Therapeutic Living Centers Solved Acute Hospital-Bed Shortage during the COVID-19 Outbreak in Daegu, Korea. J. Korean Med. Sci. 2020, 35. [CrossRef]

12. Levey, A.S.; Stevens, L.A.; Schmid, C.H.; Zhang, Y.L.; Castro, A.F., 3rd; Feldman, H.I.; Kusek, J.W.; Eggers, P.; Van Lente, F.; Greene, T.; et al. A new equation to estimate glomerular filtration rate. Ann. Intern. Med. 2009, 150, 604-612. [CrossRef] [PubMed]

13. Mehta, R.L.; Kellum, J.A.; Shah, S.V.; Molitoris, B.A.; Ronco, C.; Warnock, D.G.; Levin, A. Acute Kidney Injury Network: Report of an initiative to improve outcomes in acute kidney injury. Crit. Care 2007, 11, R31. [CrossRef] [PubMed]

14. Singer, M.; Deutschman, C.S.; Seymour, C.W.; Shankar-Hari, M.; Annane, D.; Bauer, M.; Bellomo, R.; Bernard, G.R.; Chiche, J.-D.; Coopersmith, C.M.; et al. The Third International Consensus Definitions for Sepsis and Septic Shock (Sepsis-3). JAMA 2016, 315, 801-810. [CrossRef]

15. Ranieri, V.M.; Rubenfeld, G.D.; Thompson, B.T.; Ferguson, N.D.; Caldwell, E.; Fan, E.; Camporota, L.; Slutsky, A.S. Acute respiratory distress syndrome: The Berlin Definition. JAMA 2012, 307, 2526-2533. [CrossRef]

16. Malhotra, R.; Kashani, K.B.; Macedo, E.; Kim, J.; Bouchard, J.; Wynn, S.; Li, G.; Ohno-Machado, L.; Mehta, R. A risk prediction score for acute kidney injury in the intensive care unit. Nephrol. Dial. Transplant. 2017, 32, 814-822. [CrossRef] [PubMed]

17. Basu, R.K.; Zappitelli, M.; Brunner, L.; Wang, Y.; Wong, H.R.; Chawla, L.S.; Wheeler, D.S.; Goldstein, S.L. Derivation and validation of the renal angina index to improve the prediction of acute kidney injury in critically ill children. Kidney Int. 2014, 85, 659-667. [CrossRef]

18. Wang, L.; Li, X.; Chen, H.; Yan, S.; Li, D.; Li, Y.; Gong, Z. Coronavirus Disease 19 Infection Does Not Result in Acute Kidney Injury: An Analysis of 116 Hospitalized Patients from Wuhan, China. Am. J. Nephrol. 2020. [CrossRef]

19. Zhou, F.; Yu, T.; Du, R.; Fan, G.; Liu, Y.; Liu, Z.; Xiang, J.; Wang, Y.; Song, B.; Gu, X.; et al. Clinical course and risk factors for mortality of adult inpatients with COVID-19 in Wuhan, China: A retrospective cohort study. Lancet 2020, 395, 1054-1062. [CrossRef] 
20. Finlay, S.; Bray, B.; Lewington, A.J.; Hunter-Rowe, C.T.; Banerjee, A.; Atkinson, J.M.; Jones, M.C. Identification of risk factors associated with acute kidney injury in patients admitted to acute medical units. Clin. Med. 2013, 13, 233-238. [CrossRef]

21. Bagshaw, S.M.; Sood, M.M.; Long, J.; Fowler, R.A.; Adhikari, N.K.J.; Canadian Critical Care Trials Group H1N1 Collaborative. Acute kidney injury among critically ill patients with pandemic H1N1 influenza A in Canada: Cohort study. BMC Nephrol. 2013, 14, 123. [CrossRef]

22. Hirsch,J.S.; Ng, J.H.; Ross, D.W.; Sharma, P.; Shah,H.H.; Barnett, R.L.; Hazzan, A.D.; Fishbane, S.; Jhaveri, K.D.; Abate, M.; et al. Acute kidney injury in patients hospitalized with COVID-19. Kidney Int. 2020. [CrossRef] [PubMed]

23. Silver, S.A.; Harel, Z.; McArthur, E.; Nash, D.M.; Acedillo, R.; Kitchlu, A.; Garg, A.X.; Chertow, G.M.; Bell, C.M.; Wald, R. Causes of Death after a Hospitalization with AKI. J. Am. Soc. Nephrol. 2018, 29, 1001-1010. [CrossRef] [PubMed]

24. Bagshaw, S.M.; Uchino, S.; Bellomo, R.; Morimatsu, H.; Morgera, S.; Schetz, M.; Tan, I.; Bouman, C.; Macedo, E.; Gibney, N.; et al. Septic acute kidney injury in critically ill patients: Clinical characteristics and outcomes. Clin. J. Am. Soc. Nephrol. 2007, 2, 431-439. [CrossRef] [PubMed]

25. Wang, H.E.; Muntner, P.; Chertow, G.M.; Warnock, D.G. Acute kidney injury and mortality in hospitalized patients. Am. J. Nephrol. 2012, 35, 349-355. [CrossRef]

26. Colson, P.; Rolain, J.M.; Lagier, J.C.; Brouqui, P.; Raoult, D. Chloroquine and hydroxychloroquine as available weapons to fight COVID-19. Int. J. Antimicrob. Agents 2020, 105932. [CrossRef]

27. Li, W.; Zhang, C.; Sui, J.; Kuhn, J.H.; Moore, M.J.; Luo, S.; Wong, S.K.; Huang, I.C.; Xu, K.; Vasilieva, N.; et al. Receptor and viral determinants of SARS-coronavirus adaptation to human ACE2. EMBO J. 2005, 24, 1634-1643. [CrossRef]

28. Wrapp, D.; Wang, N.; Corbett, K.S.; Goldsmith, J.A.; Hsieh, C.-L.; Abiona, O.; Graham, B.S.; McLellan, J.S. Cryo-EM structure of the 2019-nCoV spike in the prefusion conformation. Science 2020, 367, 1260-1263. [CrossRef]

29. Li, Z.; Wu, M.; Guo, J.; Yao, J.; Liao, X.; Song, S.; Han, M.; Li, J.; Duan, G.; Zhou, Y.; et al. Caution on Kidney Dysfunctions of 2019-nCoV Patients. MedRxiv 2020. [CrossRef]

30. Farkash, E.A.; Wilson, A.M.; Jentzen, J.M. Ultrastructural Evidence for Direct Renal Infection with SARS-CoV-2. J. Am. Soc. Nephrol. 2020. [CrossRef]

31. Su, H.; Yang, M.; Wan, C.; Yi, L.-X.; Tang, F.; Zhu, H.-Y.; Yi, F.; Yang, H.-C.; Fogo, A.B.; Nie, X.; et al. Renal histopathological analysis of 26 postmortem findings of patients with COVID-19 in China. Kidney Int. 2020. [CrossRef]

32. Peerapornratana, S.; Manrique-Caballero, C.L.; Gómez, H.; Kellum, J.A. Acute kidney injury from sepsis: Current concepts, epidemiology, pathophysiology, prevention and treatment. Kidney Int. 2019, 96, 1083-1099. [CrossRef] [PubMed]

33. Han, S.J.; Lee, H.T. Mechanisms and therapeutic targets of ischemic acute kidney injury. Kidney Res. Clin. Pract. 2019, 38, 427-440. [CrossRef] [PubMed]

34. Gautret, P.; Lagier, J.C.; Parola, P.; Hoang, V.T.; Meddeb, L.; Mailhe, M.; Doudier, B.; Courjon, J.; Giordanengo, V.; Vieira, V.E.; et al. Hydroxychloroquine and azithromycin as a treatment of COVID-19: Results of an open-label non-randomized clinical trial. Int. J. Antimicrob. Agents 2020. [CrossRef] [PubMed] 